\title{
Measurements of the Characteristics of Transparent Material Using Digital Holography
}

\author{
Ding Yu, ${ }^{1}$ Shang Wenbin, ${ }^{2}$ Yang Hong, ${ }^{3}$ and Yang Yan $^{2}$ \\ ${ }^{1}$ Chongqing City Management College, Shapingba District, Chongqing 401331, China \\ ${ }^{2}$ Mechanical Engineering, Chongqing University of Technology, No. 69 Hongguang Road, Banan District, Chongqing 400054, China \\ ${ }^{3}$ Key Laboratory of Advanced Manufacturing Technology for Automobile Parts, Ministry of Education, No. 69 Hongguang Road, \\ Banan District, Chongqing 400054, China
}

Correspondence should be addressed to Yang Yan; 40129784@qq.com

Received 31 July 2013; Revised 28 September 2013; Accepted 28 September 2013

Academic Editor: Xing Chen

Copyright (C) 2013 Ding Yu et al. This is an open access article distributed under the Creative Commons Attribution License, which permits unrestricted use, distribution, and reproduction in any medium, provided the original work is properly cited.

Digital holography is applied to measure the characteristics of transparent material. A digital hologram recording system to measure the surface of transparent material was established, and the digital holograms of transparent object were obtained in high quality. For postprocessing of hologram, the least-squares phase unwrapping algorithm was used in phase unwrapping, and the phase reconstruction image of transparent object was obtained. The information of material surfaces was measured and the characteristic was presented in 3D visualization. The validation experiment was conducted by NanoMap 500LS system; the results of validation experiment are well satisfied with the measurement by digital holography, which proved the feasibility of digital holographic technology as a good measurement tool for transparent material.

\section{Introduction}

In 1948 , Gabor $[1,2]$ proposed holography, which can be used for reconstructing amplitude and phase of wave field. With the fast development of computer technology, recording and reconstruction are made possible by means of computer. Subsequently, Goodman and Lawrence [3] developed digital holography in 1960s.

Digital holography is a new image processing technology that uses a high-resolution charge-coupled device (CCD) camera for hologram recording and image reconstruction with a numerical method by a computer [4]. Compared with traditional holography, which uses a photographic plates as recording media, digital holography has a significant improvement; that is to say, it does not need complicated chemical processing [5], thus, adding more flexibility and improving the efficiency of the holographic process. In the past few years, with modern computer technology and CCD technology developing rapidly, digital holography has been established as an important scientific means applied in metrology [6], surface measurement, deformation measurement [7], and more recently biological microscopy [8-10].

In general, the objects in applications of digital holography are not transparent, so the holograms were captured by the reflection of the object [11-13]. In this paper, we adopt the method of transmission to generate the hologram.

Noncontact surface measurement techniques are very important in many fields, such as science and engineering. It is very helpful to control the products quality and make the appropriate diagnosis. Digital holographic imaging as a new image processing technology, combined with optical holography and digital image processing of computer, is widely used in the field of surface measurements of objects. In addition to classical roughness values $R_{a}, R_{t}$, and so forth, digital holography can also make full-field measurements of 3D surface material. Digital holographic measurement can measure the surface of the object with some important characteristics, such as no damage, high resolution, and noncontact fast processing. 


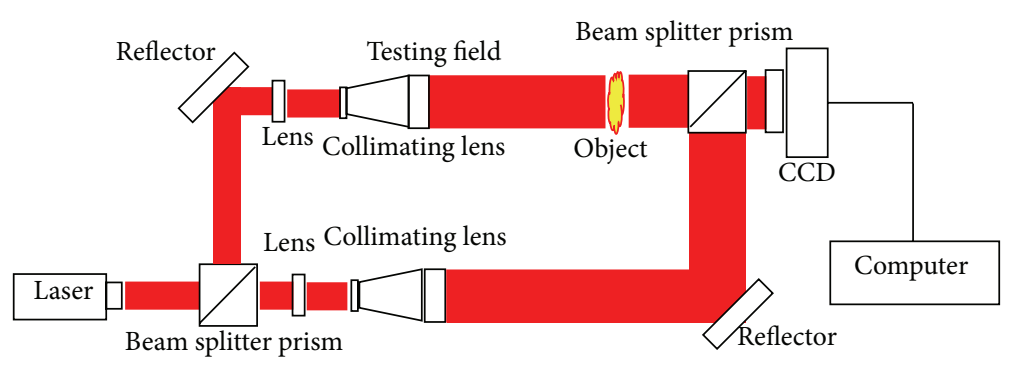

FIGURE 1: Off-line recording of digital hologram.

Compared with other image processing and measuring techniques, digital holography has a number of important advantages [4]. Firstly, it does not require much optical equipment, especially changing the hologram plates. Secondly, it captures the image with noncontact, nondestructive, and fast, height accuracy, sensitivity and resolution. Thirdly, there is no complex chemical processing in digital holography, so reproducing hologram and reconstructing the hologram are quicker than traditional optical holography. Fourthly, computer image processing allows to process the errors and noise so as to revise additional optical phase for improving the quality of the reconstructed image.

Holographic interferometry (HI) is a procedure which enables static and dynamic displacements of objects with optically rough surfaces to be measured with optical interferometer accuracy $[4,14]$. Then, in digital holography, we can accurately measure the slight deformation of the objects by calculating the change of the information recorded by digital holograms [4]. In this paper, through digital holography and image processing, we can measure the height of the material surface. Otherwise, by recording the hologram, the phase diagram can be obtained and the surface of material can be measured quickly and accurately, making the threedimensional visualization possible.

\section{General Principles of Digital Holography}

2.1. Recording of Digital Hologram. Digital holographic recording optical path and the traditional optical holography are consistent; only replace holographic plate with a CCD camera as the recording medium. The concept of outline digital hologram recording is shown in Figure 1.

The object wave $O(x, y)$ and the reference wave $R(x, y)$ interference occur to form a hologram on the CCD surface. In the hologram plane, the recorded intensity distribution $I_{H}(x, y)$ can be printed as follows [4]:

$$
H(x, y)=|O|^{2}+|R|^{2}+R O^{*}+R^{*} O
$$

where $R O^{*}$ and $R^{*} O$ represent the conjugation image and the original image, respectively.

So the intensity distribution of the recorded hologram by CCD is written as

$$
H(m, n)=H(x, y) \operatorname{rect}\left(\frac{x}{L_{x}}, \frac{y}{L_{y}}\right) \operatorname{comb}\left(\frac{x}{\Delta_{x}}, \frac{y}{\Delta_{y}}\right) \text {. }
$$

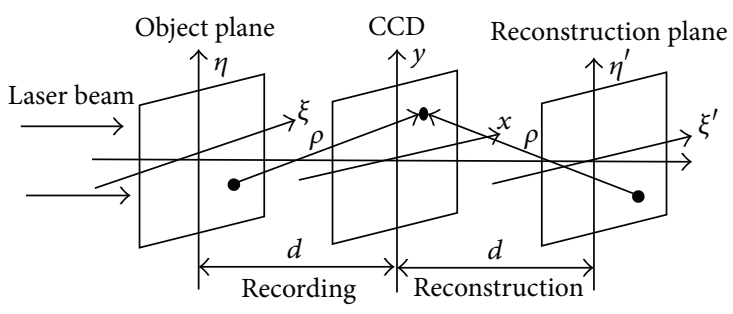

FIGURE 2: Coordinate system for image reconstruction.

The light intensity is collected by the data acquisition card and quantity. The 3D information of object is saved by the computer and formed as a digital hologram.

2.2. Reconstruction of Digital Hologram. In traditional holography, the reconstruction is carried out by means of illumination of the hologram intensity with the reference wave. A virtual image and a real image of the object are reconstructed. The digital hologram simulates the traditional optical reproduction processes, and its numerical reconstruction can be gained by computer.

The laser beam goes through the object and the part of the beam diffracted by objects and reaching the recording surface is considered the object beam, while the beam from the laser arriving without any distortion is considered as the reference beam. Two beams superposition create an interference pattern on the CCD sensor. This diffraction can be described by the Fresnel-Kirchhoff integral as [4]

$$
\begin{aligned}
& R\left(\xi^{\prime}, \eta^{\prime}\right) \\
& \quad=\frac{i}{\lambda} \iint_{-\infty}^{\infty} h(x, y) E_{R}(x, y) \frac{\exp (-i(2 \pi / \lambda) \rho)}{\rho} d x d y,
\end{aligned}
$$

with

$$
\rho=\sqrt{\left(\xi^{\prime}-x\right)^{2}+\left(\eta^{\prime}-y\right)^{2}+d^{2}} .
$$

The coordinates in (3) and (4) are illustrated in Figure 2. $R\left(\xi^{\prime}, \eta^{\prime}\right)$ is the wave fields of the reconstruction image and $h(x, y)$ is the hologram function. The $\rho$ is the distance between a point in the hologram plane and the corresponding point in the reconstruction plane, $d$ is the distance between two adjacent planes, and $\lambda$ is the wavelength.

Different numerical reconstruction algorithms have been proposed, such as Fresnel approximation algorithm and 
convolution approach algorithm [4]. The resolution of the reconstruction image by convolution approach algorithms is much better than that by the Fresnel approximation algorithm in [10]. Therefore, the convolution approach algorithm is adopted in this paper and the mathematical expression of the convolution approach algorithm is defined as follows:

$$
\begin{aligned}
& R\left(\xi^{\prime}, \eta^{\prime}\right) \\
& =F^{-1}\{F[h(x, y)] \\
& \cdot F\left[\frac { i } { \lambda } \left(\operatorname { e x p } \left[-i \frac{2 \pi}{\lambda}\right.\right.\right. \\
& \left.\left.\times \sqrt{d^{2}+\left(x-\xi^{\prime}\right)^{2}+\left(y-\eta^{\prime}\right)^{2}}\right]\right) \\
& \left.\left.\times\left(\sqrt{d^{2}+\left(x-\xi^{\prime}\right)^{2}+\left(y-\eta^{\prime}\right)^{2}}\right)^{-1}\right]\right\},
\end{aligned}
$$

where $F[]$ and $F^{-1}[]$ are the Fourier transform and the inverse Fourier transform, respectively.

In this paper, we use numerical simulation hologram to check the ability of our arithmetic for determination of the focal plane. For numerical simulation reconstruction of holograms, the Fresnel and convolution arithmetic based on the reconstruction equations has been used well in practice. The convolution arithmetic producing good holograms and reconstruction images and high quality of holograms does not change with different object distances. Therefore, convolution arithmetic will be importantly explained in this paper.

In Figure 2, the left and the right part are the same, which means the formula for reconstruction image from a hologram is the same as that for the generation of the hologram from the object image. Thus, after change of some parameters in (3), we will obtain the new hologram function as follows:

$$
h(x, y)=\frac{i}{\lambda} \iint_{-\infty}^{\infty} O(\xi, \eta) E_{R}(\xi, \eta) \frac{\exp (-i(2 \pi / \lambda) \rho)}{\rho} d \xi d \eta
$$

Because the form of (6) is the same as that of (3), the convolution algorithm can also be used to obtain the hologram function, $h(x, y)$, from the object function, $O(\xi, \eta)$. Then the replacement of (3) and (6) can be rewritten in the form of the superposition integral as follows:

$$
h(x, y)=\iint_{-\infty}^{\infty} O(\xi, \eta) g(\xi, \eta, x, y) d \xi d \eta,
$$

with

$$
\begin{aligned}
& g(\xi, \eta, x, y) \\
& \quad=\frac{i}{\lambda} \frac{\exp \left[-i(2 \pi / \lambda) \sqrt{d^{2}+(\xi-x)^{2}+(\eta-y)^{2}}\right]}{\sqrt{d^{2}+(\xi-x)^{2}+(\eta-y)^{2}}} .
\end{aligned}
$$

This superposition integral can be considered as a convolution. So, (7) will become

$$
\begin{aligned}
& h(x, y) \\
& =F^{-1}\{F[O(\xi, \eta)] \\
& \cdot F\left[\frac{i}{\lambda}\left(\exp \left[-i \frac{2 \pi}{\lambda} \sqrt{d^{2}+(\xi-x)^{2}+(\eta-y)^{2}}\right]\right)\right. \\
& \left.\left.\quad \times\left(\sqrt{d^{2}+(\xi-x)^{2}+(\eta-y)^{2}}\right)^{-1}\right]\right\} .
\end{aligned}
$$

Equation (9) is used for numerical simulation of holograms by the convolution algorithm. Based on this equation, we can get the hologram function, $h(x, y)$, equivalent to some object image function, $O(\xi, \eta)[4]$.

\section{Theories of Surface Measurements by Digital Holography}

3.1. General Principles. As the material surfaces are uneven, when irradiated by the laser, there will appear a different phase on the material surfaces. In Section 2, after recording and reconstruction of digital hologram, the intensity and phase distribution of the reconstructed image can be obtained by [4]

$$
\begin{gathered}
I(\xi, \eta)=|R(\xi, \eta)|^{2}=\operatorname{Re}^{2}|R(\xi, \eta)|+\operatorname{Im}^{2}|R(\xi, \eta)|, \\
\Phi(\xi, \eta)=\arctan \frac{\operatorname{Im}|R(\xi, \eta)|}{\operatorname{Re}|R(\xi, \eta)|}
\end{gathered}
$$

where $\operatorname{Re}|R(\xi, \eta)|$ and $\operatorname{Im}|R(\xi, \eta)|$ denote the real and imaginary parts of the object complex amplitude, respectively.

3.2. Phase Unwrapping. Phase distribution value obtained by (11) is limited in the range of $(-\pi,+\pi)$ for the theory of the arctan function, so also through phase unwrapping, the accurate phase information can be obtained [4]. Phase unwrapping is a method applied to wrapped phase images to remove the $2 \pi$ incoherence embedded within the phase diagram. It detects a $2 \pi$ phase jump and adds or subtracts an integer offset of $2 \pi$ to adjoining pixels following that phase jump based on a tolerance mechanism, thus, retrieving the continuous form of the phase map. The simulation of phase unwrapping process is illustrated in Figures 3(a) and 3(b).

Phase unwrapping is a technique which can generate a continuously phase distribution. It constitutes essential parts of optical metrology by heterodyne techniques. In surface measurement by digital holographic, the phase unwrapping is a key technique. Numerous phase unwrapping algorithms have been proposed in the past several years $[15,16]$. Two types of strategy have been developed to solve the 


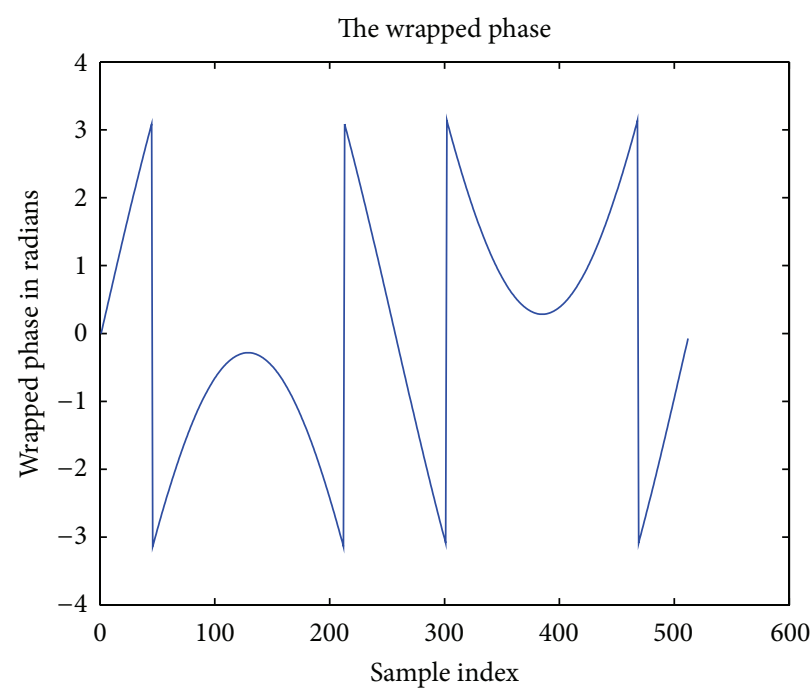

(a)

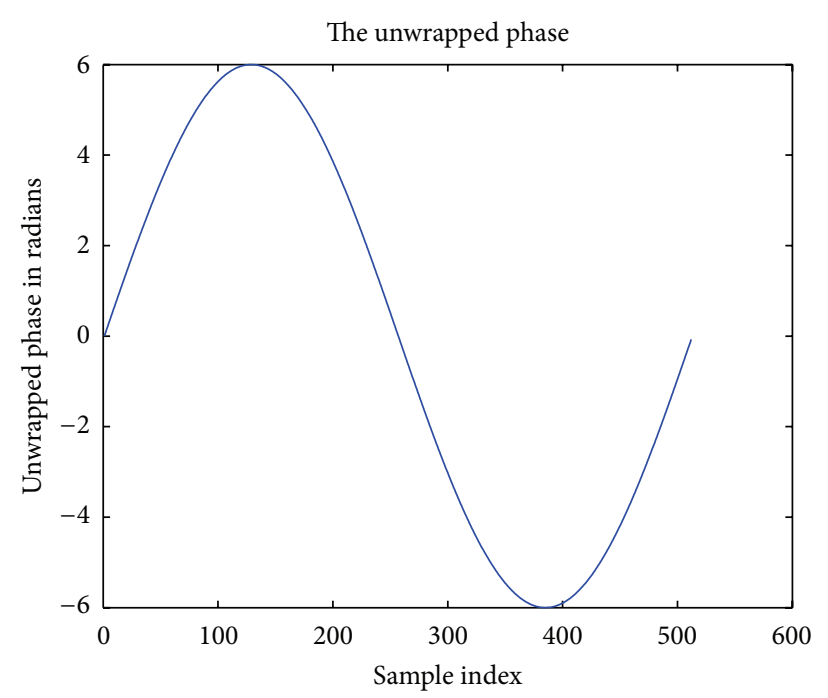

(b)

FIGURE 3: Phase unwrapping: (a) wrapped phase and (b) continuous phase.

phase unwrapping problem: path-following and minimumnorm methods. Path-following methods include Goldstein's branch cut algorithm [17], and minimum spanning tree algorithm [18]. Minimum-norm methods contain least-squares phase unwrapping algorithm [19], minimum Lp-norm phase unwrapping algorithm [16], and so on.

In this paper, the continuous phase image is acquired by least-squares phase unwrapping algorithm without weighing. The discrete cosine transforms (DCT) is used to solve the discrete Poisson equation in this method. The least-square solution of the unwrapped phase and the expanded phase is obtained.

3.3. The Height Distribution of Surface. As mentioned above, after the phase unwrapping with computer, the phase distribution $\Phi(\xi, \eta)$ can be simply converted into the height distribution $h(\xi, \eta)$ on the material surface. Then, the continuous phase distribution can be used for measurements of material surface. So the height of the material surface $h(\xi, \eta)$ can be calculated by [4]

$$
h(\xi, \eta)=\frac{\lambda}{4 \pi} \Phi(\xi, \eta),
$$

where $\lambda$ is the wavelength.

The surface of homogenous optical properties was measured in the light of the reconstructed phase-contrast image [19], which showed the phase changes corresponding to optical path was less than $10 \mathrm{~nm}$. In this paper, the target we choose is much bigger than $10 \mathrm{~nm}$.

\section{Experiments and Results}

4.1. Optical Experiment. Since the height of the target surface is uniformly distributed, in this experiment a test target is used as the target surface. The optical experimental setup for recording test target (USFA 1950) holograms is shown in

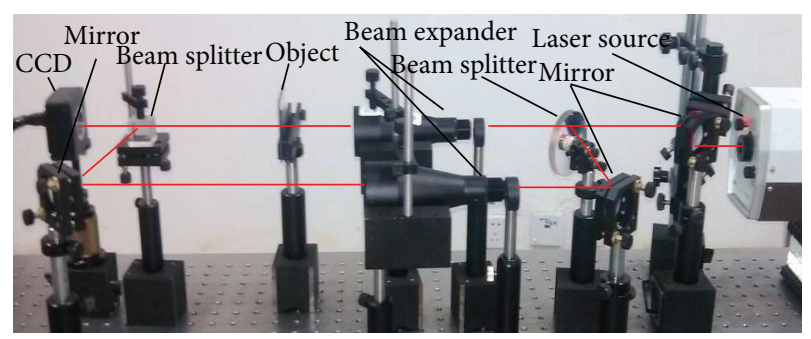

FIGURE 4: Experimental setup for recording test target holograms.

Figure 4. The experimental conditions are listed as follows: the pixel number $N$ is equal to 1024; the pixel size of the CCD camera is $5.2 \mu \mathrm{m}$; the wavelength $\lambda$ is equal to $632.8 \mathrm{~nm}$. The laser with a maximum output power of $6 \mathrm{~W}$ is adopted.

As shown in Figure 4, the input He-Ne laser is divided into two parts by a beam splitter (BS): one beam goes through the test target as the object beam and another beam is expanded as the reference beam. The object and reference beams have an interference with BS, the hologram is recorded by a CCD detector, and then the image information is sent to the computer by a collection.

Figure 5 shows the recording of digital hologram by computer. The recording distance between the object and the CCD sensors is set to be $78 \mathrm{~mm}$. Figure 6 shows the reconstruction intensity image of the test target. Then the height distribution $h(\xi, \eta)$ can be obtained from (11) and (12). Through the powerful drawing function of MATLAB, the height distribution of the material surface can be displayed in the form of 3D; it was shown in Figure 7. 1002 points on material surface were measured in experiment, and the height distribution information is illustrated in Figure 8 and Table 1.

4.2. Validation Experiment. The verification experimental equipment uses NanoMap 500LS which is produced by AEP 


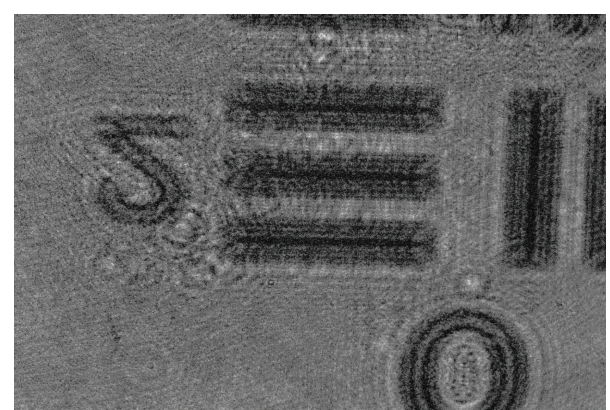

FIGURE 5: Recording of digital hologram.

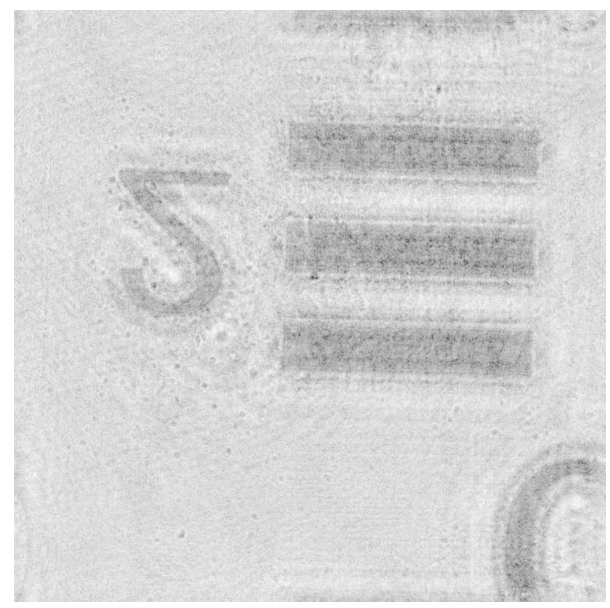

FIGURE 6: Reconstruction of digital hologram.

technology, and the composition of the equipment is the same as the one in Figure 9 [20]. NanoMap has many features: (a) seamless integration of conventional contact profile meter and scanning probe microscope (SPM) technology, (b) dual mode operation (tip scan and stage scan) optimized for small areas, (c) 3D mapping as well as long range profiling and 3D long scan range up to $150 \mathrm{~mm} \times 150 \mathrm{~mm}$, (d) stage scan by using high grade optical reference flat, (e) wide vertical range with high accuracy as a result of the dual optical and up to $0.1 \mathrm{~nm}$ vertical resolution with fine sensor, (f) constant contact forces suitable by software and automatic sample positioning with $X Y$ motorized stage. The NanoMap 500LS measurement flow chart is shown in Figure 10.

After loading the test target onto the stage and setting up scan parameters, we can get the scan curve shown in Figure 11. We process the data and curve fitting using MATLAB and obtain the result shown in Figure 12. The height distribution information is illustrated in Figure 13 and the height dates in Table 2. From Figures 12 and 13, we can see so many more numbers whose values are smaller than zero, the reason for which is that the protruding part is less than the hollow one in the part we scan. In Figure 13 and Table 2, the average measurement result of the target surface by NanoMap 500LS is $69.9 \mathrm{~nm}$.

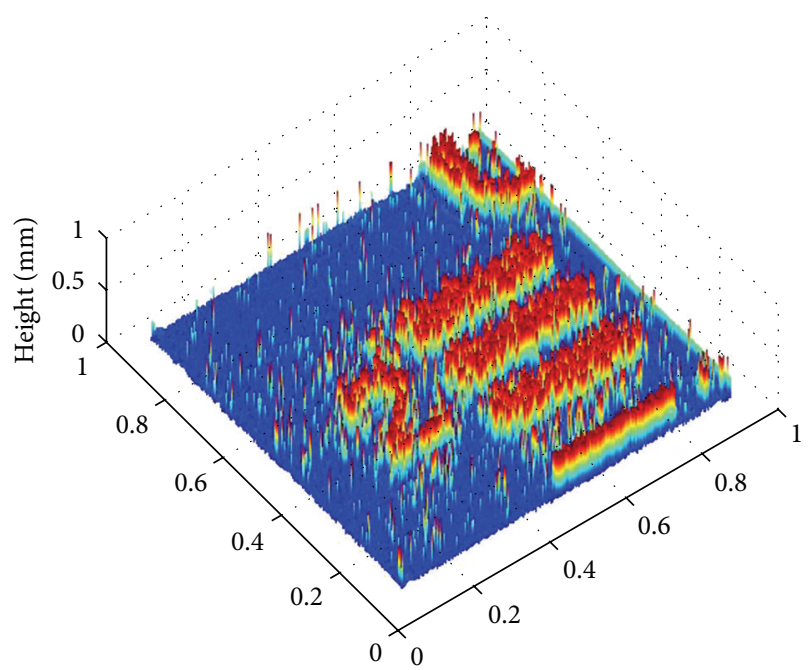

FIGURE 7: The reconstructed height distribution.

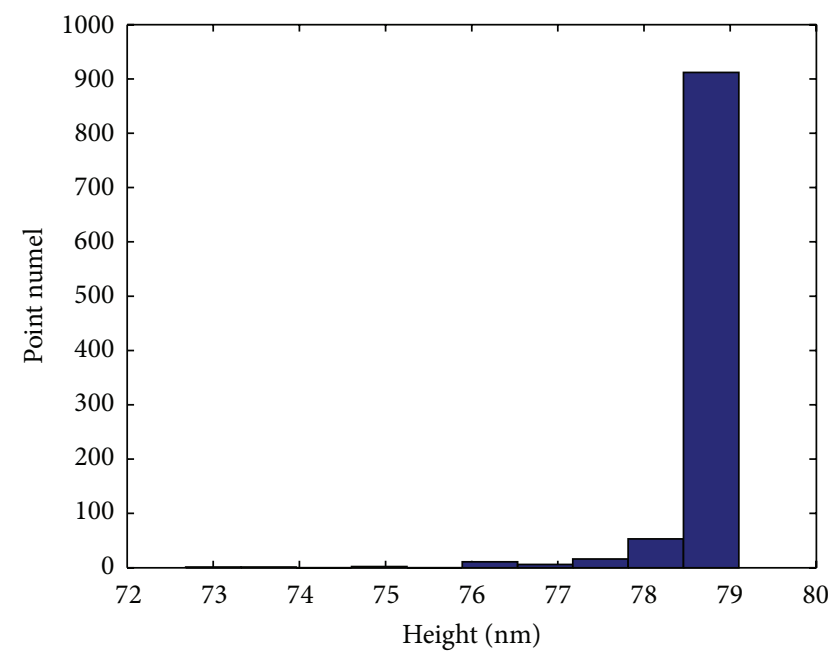

FIGURE 8: Height distribution.

TABLE 1: Data of heights.

\begin{tabular}{lccc}
\hline Height & Min & Max & Average \\
\hline Value $(\mathrm{nm})$ & 72.7 & 79.1 & 78.8 \\
\hline
\end{tabular}

TABLE 2: Data of heights.

\begin{tabular}{lccc}
\hline Height & Min & Max & Average \\
\hline Value $(\mathrm{nm})$ & 60.4 & 87.6 & 69.9 \\
\hline
\end{tabular}

4.3. Experimental Results. Tables 1 and 2 show the measurement results of the target surface by digital holography and NanoMap500Ls. The comparison of two methods is shown in Table 3. The relative error can be obtain as follows: $8.9 / 69.9=12.7 \%$. The digital holography can serve as an effective measurement method used in engineering. 
TABLE 3: Comparison of the measurement results by two methods.

\begin{tabular}{lcccc}
\hline Methods & Digital holography $(\mathrm{nm})$ & NanoMap 500Ls $(\mathrm{nm})$ & Difference $(\mathrm{nm})$ & Relative error $(\%)$ \\
\hline Value & 78.8 & 69.9 & 8.9 & 12.7 \\
\hline
\end{tabular}

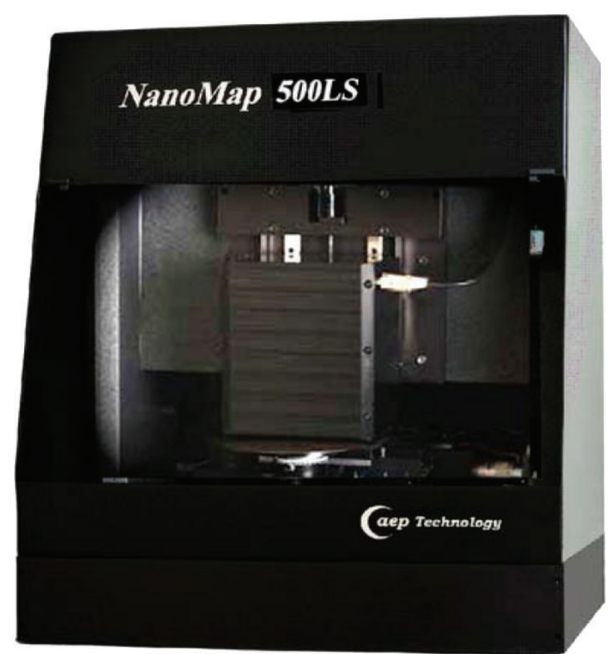

Figure 9: NanoMap 500LS.

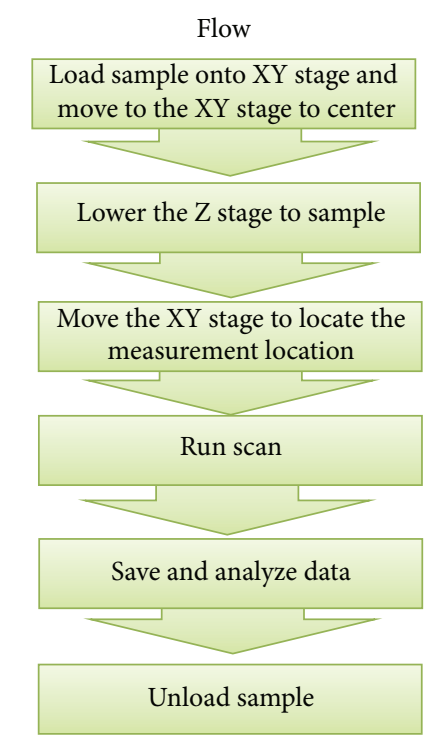

FIGURE 10: Flow chart for taking a profile measurement.

\section{Conclusions}

In this paper, we have presented the digital holography for measuring the material surface as an important technique. The principle of surface measurement by digital holography is analyzed. In this paper, we use a target as a test target. By recording and reconstruction hologram, we obtain the phase and height distribution of the object surface by noise, phase unwrapping, and so forth. Then we use NanoMap 500LS 3D profilometer as validation experiment. Comprised

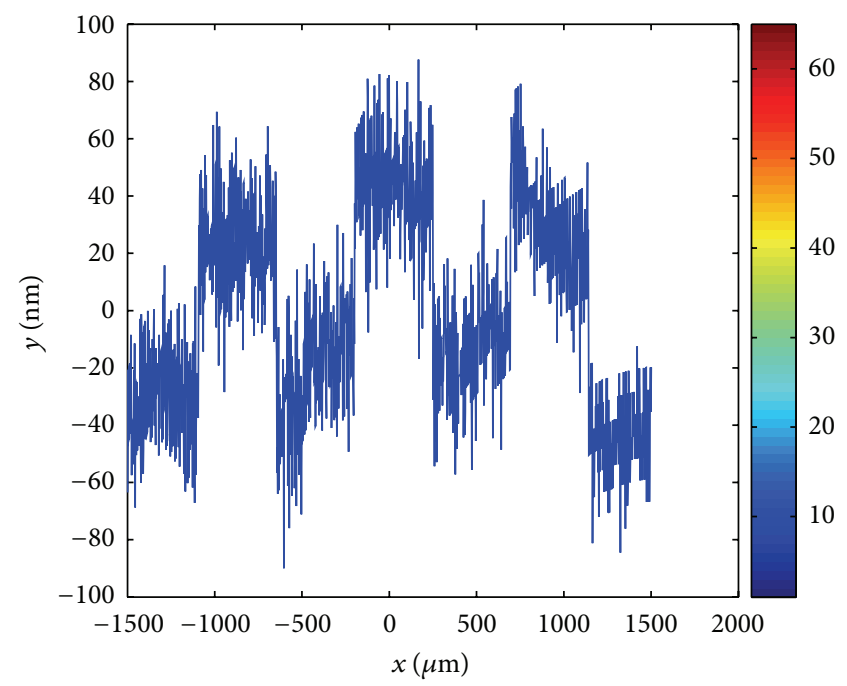

FIGURE 11: The scan curve.

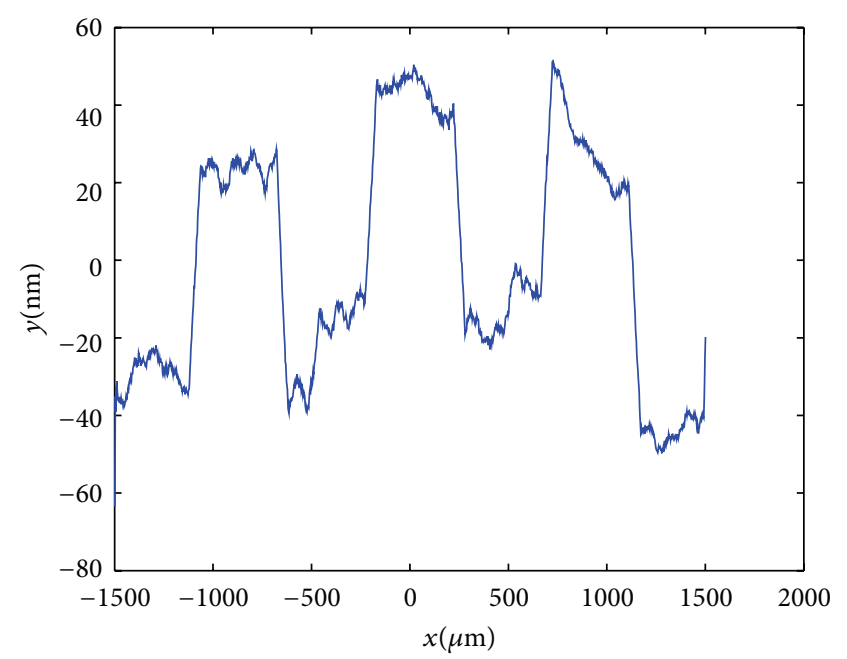

FIgURE 12: The fitting curve.

of contact profilometer, the results of two measurement methods do not make much difference. However, digital holography measurement method has the larger advantage. Digital holographic measurement can obtain the surface of objects with no damage, high resolution, noncontact, and fast processing characteristics. And also, any measurement system has measuring error. Future research direction is to solve the measurement errors which exist. 


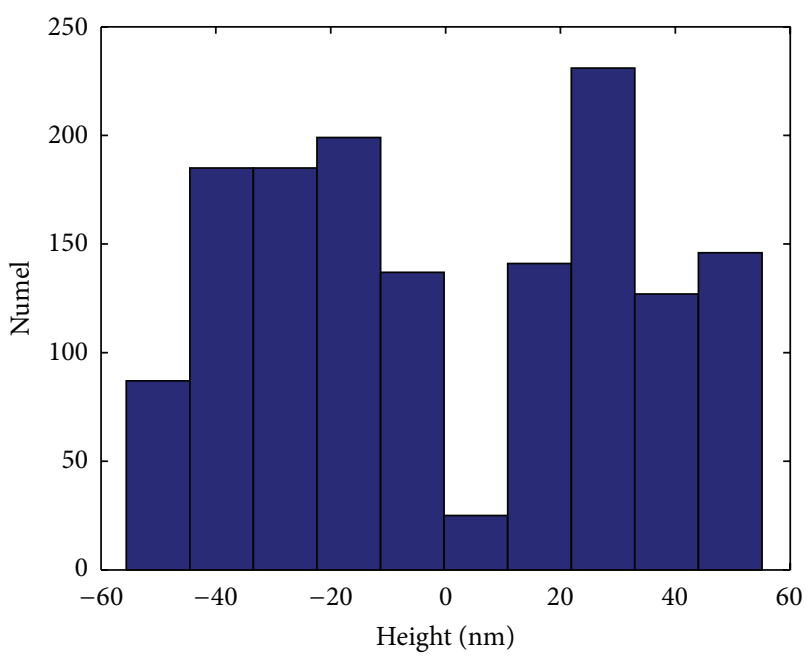

FIGURE 13: Height distribution.

\section{Acknowledgments}

This work was supported by the National Natural Science Foundation of China (11272368) and the Natural Science Foundation of CSTC (cstc2013yykfB0198).

\section{References}

[1] D. Gabor, "A new microscopic principle," Nature, vol. 161, no. 4098, pp. 777-778, 1948.

[2] D. Gabor, "Microscopy by reconstructed wavefronts: 2," Proceedings of the Royal Society, vol. 64, pp. 449-469, 1951.

[3] J. W. Goodman and R. W. Lawrence, "Digital image formation from electronically detected holograms," Applied Physics Letters, vol. 11, no. 3, pp. 77-79, 1967.

[4] Y. Yang, B.-S. Kang, and Y.-J. Choo, "Application of the correlation coefficient method for determination of the focal plane to digital particle holography," Applied Optics, vol. 47, no. 6, pp. 817-824, 2008.

[5] Y. Yang and B.-S. Kang, "Experimental validation for the determination of particle positions by the correlation coefficient method in digital particle holography," Applied Optics, vol. 47, no. 32, pp. 5953-5960, 2008.

[6] L. Xu, X. Peng, J. Miao, and A. K. Asundi, "Studies of digital microscopic holography with applications to microstructure testing," Applied Optics, vol. 40, no. 28, pp. 5046-5051, 2001.

[7] G. Pedrini and H. J. Tiziani, "Quantitative evaluation of twodimensional dynamic deformations using digital holography," Optics and Laser Technology, vol. 29, no. 5, pp. 249-256, 1997.

[8] Y. Yang and B. Kang, "Measurements of the characteristics of spray droplets using in-line digital particle holography," Journal of Mechanical Science and Technology, vol. 23, no. 6, pp. 16701679, 2009.

[9] G. Popescu, L. P. Deflores, J. C. Vaughan et al., "Fourier phase microscopy for investigation of biological structures and dynamics," Optics Letters, vol. 29, no. 21, pp. 2503-2505, 2004.

[10] P. Marquet, B. Rappaz, P. J. Magistretti et al., "Digital holographic microscopy: a noninvasive contrast imaging technique allowing quantitative visualization of living cells with subwavelength axial accuracy," Optics Letters, vol. 30, no. 5, pp. 468-470, 2005.

[11] A. Ettemeyer, "Applications of digital holography to microstructures," in Proceedings of the SAE World Congress 2009, Detroit, Mich, USA, April 2009.

[12] S. Seebacher, W. Osten, and W. P. O. Jüptner, "Measuring shape and deformation of small objects using digital holography," in Proceedings of SPIE, pp. 104-115, July 1998.

[13] B. Bowe and V. Toal, "White light interferometric surface profiler," Optical Engineering, vol. 37, no. 6, pp. 1796-1799, 1998.

[14] T. Kreis, Handbook of Holographic Interferometry, Wiley-VCH, 2005.

[15] R. M. Goldstein, H. A. Zebker, and C. L. Werner, "Satellite radar interferometry: two-dimensional phase unwrapping," Radio Science, vol. 23, no. 4, pp. 713-720, 1988.

[16] D. C. Ghiglia and M. D. Pritt, Two-Dimensional Phase Unwrapping: Theory, Algorithms, and Software, John Wiley \& Sons, Hoboken, NJ, USA, 1998.

[17] R. M. Goldstein, H. A. Zebker, and C. L. Werner, "Satellite radar interferometry: two-dimensional phase unwrapping," Radio Science, vol. 23, no. 4, pp. 713-720, 1988.

[18] J. Schöner, A. Ettemeyer, U. Neupert, H. Rottenkolber, C. Winter, and P. Obermeier, "New approaches in interpreting holographic images," Optics and Lasers in Engineering, vol. 14, no. 4-5, pp. 283-291, 1991.

[19] D. Kerr, G. H. Kaufmann, and G. E. Galizzi, "Unwrapping of interferometric phase-fringe maps by the discrete cosine transform," Applied Optics, vol. 35, no. 5, pp. 810-816, 1996.

[20] AEP Technology, "NanoMap 500LS 3D profilometer User's Manual," Revision B, 2009. 

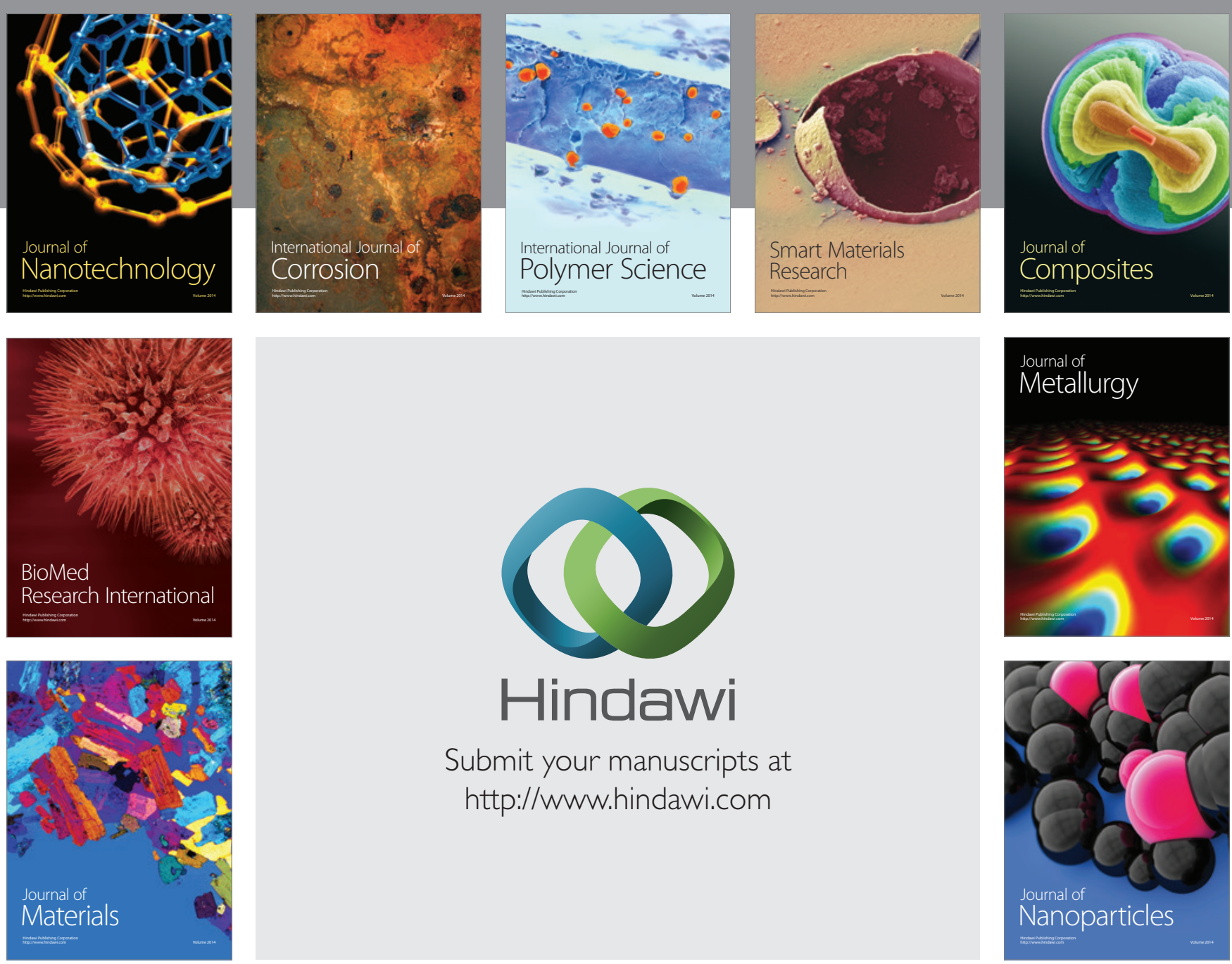

Submit your manuscripts at http://www.hindawi.com
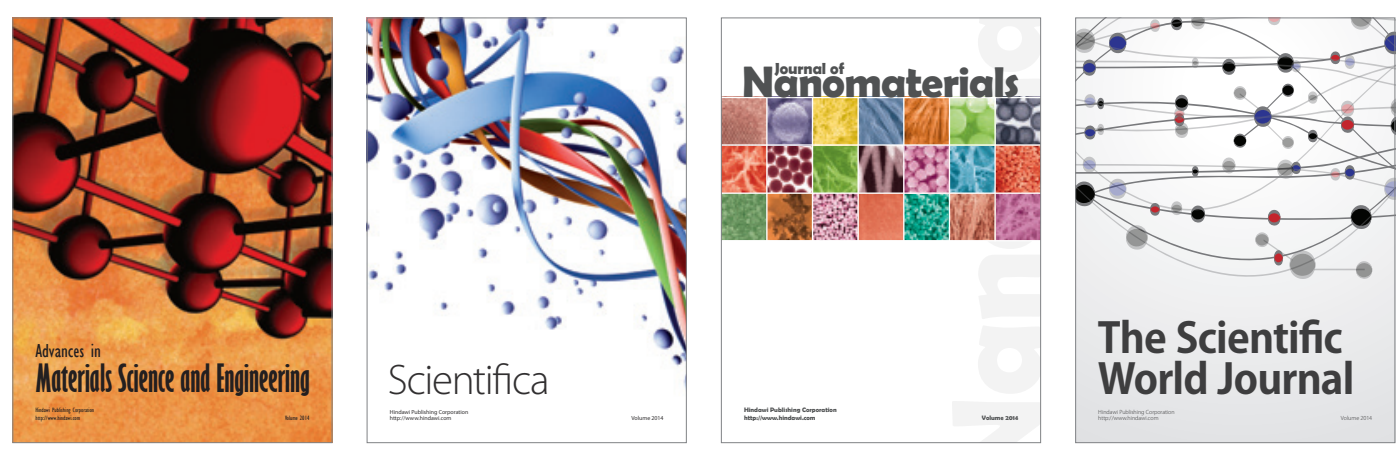

\section{The Scientific World Journal}
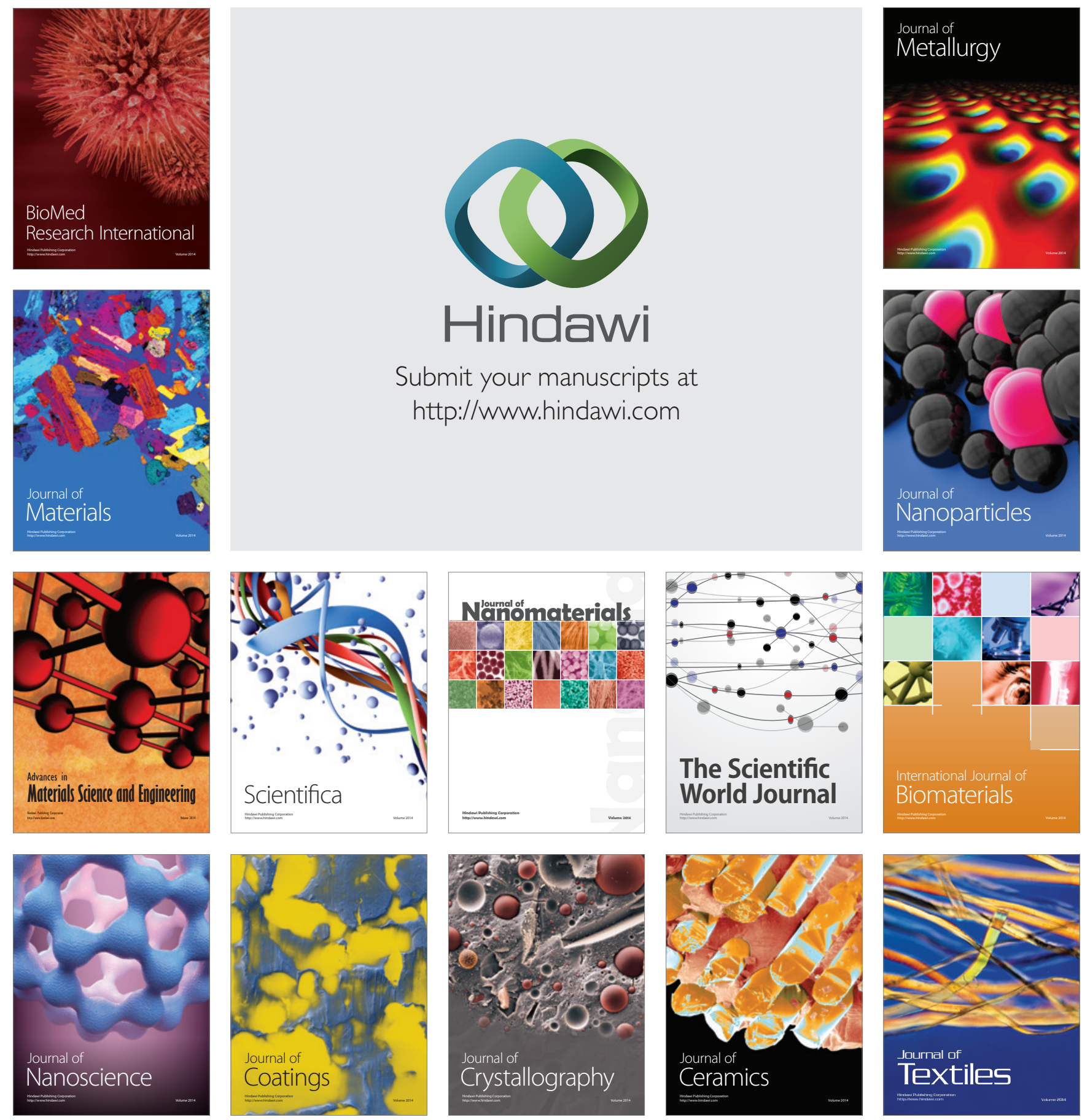